“ (C) 2018 IEEE. Personal use of this material is permitted. Permission from IEEE must be obtained for all other uses, in any current or future media, including

reprinting/republishing this material for advertising or promotional purposes, creating new collective works, for resale or redistribution to servers or lists, or reuse of any copyrighted component of this work in other works." 


\title{
State of Art Technologies for Development of High Frequency Transformers with Advanced Magnetic Materials
}

\author{
Pejush Chandra Sarker, Md. Rabiul Islam, Senior Member, IEEE, Youguang Guo, Senior Member, \\ IEEE, Jianguo Zhu, Senior Member, IEEE, and Haiyan Lu, Senior Member, IEEE
}

\begin{abstract}
With the development of advanced soft magnetic materials of high saturation flux density and low specific core loss and semiconductor power devices, the high frequency transformer (HFT) has received significant attentions in recent years for its widespread emerging applications. The optimal design of high power density HFTs for high performance energy conversion systems, however, is a multiphasic problem that needs special considerations on various aspects such as core material selection, minimization of parasitic components and thermal management. This paper presents a comprehensive review on advancement of soft magnetic materials for high power density magnetic devices and advanced technologies for characterizations and optimal design of HFTs. The future research and development trends are also discussed.
\end{abstract}

Index Terms - High frequency transformer, amorphous and nanocrystalline magnetic materials, modeling of parasitic components, characterization.

\section{INTRODUCTION}

$\mathrm{R}$ EDUCTION of size and weight and improvement of energy efficiency of power devices are the key research objectives in recent decades. The high temperature superconducting (HTS) cable technology enables people to design compact, lightweight and efficient power devices [1]-[3]. Scientists all over the world are pushing the HTS technology for developing large scale electrical machines (more than $10 \mathrm{MW}$ ) [4], [5], [6]. It is now obvious that HTS cable can reduce the transmission line weight and power loss significantly [7], [8], [9]. On the other hand, increasing the operating frequency can greatly reduce the volume and weight of an electro-magnetic device to achieve high power density. In recent years, various types of high frequency transformers (HFTs) have been developed for emerging applications such as solid state transformers (SSTs) and power electronic converters in smart grids, renewable energy, energy storage, and traction systems [10]-[12]. In the wide spread distributed renewable power generation and the associated management systems, the HFT is an enabling component with a high possibility to substitute the conventional

Manuscript submission for review, September 19, 2018.

P. C. Sarker, Y. G. Guo, and H. Y. Lu are with Faculty of Engineering and Information Technology, University of Technology Sydney, Broadway NSW 2007, Australia. E-mails: PejushChandra.Sarker@ student.uts.edu.au, Youguang.Guo-1@uts.edu.au, Haiyan.Lu@uts.edu.au.

M. R. Islam is with the School of Electrical, Computer and Telecommunications Engineering, Faculty of Engineering and Information Sciences, University of Wollongong, Wollongong, NSW 2522, Australia. Emails: rabiulbd@ hotmail.com, mrislam@uow.edu.au. low frequency transformer. According to the Massachusetts Institute of Technology Review in 2010, the SST has been considered as one of the highly growing technologies [12]. Using high power density HFTs and semiconductor devices, the SST can have much smaller volume and lower weight than those of the conventional low frequency transformer. While most HFTs are dry types, for high power range water cooling can sometimes be employed and thus the periodic replacement of oil or dielectric liquid used for cooling and insulation in the conventional transformer can be avoided.

Recently, extensive research has been carried out on design and characterization of HFTs. A review of SSTs and different configurations in the field of power distribution was presented in [12] with a focus on the SST applications. In [13]-[15], different topologies in the field of power conversion between medium ac voltage and low dc voltage, integration of renewable energy, medium voltage grid to lower voltage grid, and high voltage pulsed power supply sectors were proposed and analyzed. In [16] and [17], the properties of different soft magnetic materials were investigated. The core loss measurement methods and their empirical expressions are necessary for characterization of HFTs. Various experimental techniques and models have been proposed to measure core loss in [18]-[22]. The winding parasitic elements such as resistance, leakage inductance and stray capacitance can significantly affect the performance of an HFT. Different experimental, analytical and simulation methods have been proposed to measure and predict the parasitic elements [23]-[26]. Since the loss is dissipated as heat, proper thermal management is required to operate the HFTs safely. In [10], [27] and [28], the authors proposed different thermal management techniques for the HFTs. However, the HFT technology is still facing many challenges such as high core loss due to higher frequency operation and large volume due to low saturation flux densities of core materials [29]-[31]. To avoid the high core loss in the conventional core material, e.g. silicon iron at high operating frequency, advanced soft magnetic core materials with high saturation flux density and low specific core loss such as

J. G. Zhu is with the School of Electrical and Information Engineering, The University of Sydney, NSW 2006, Australia, E-mail: jianguo.zhu@sydney.edu.au.

Color versions of one or more of the figures in this paper are available online at http://ieeexplore.ieee.org.

Digital Object Identifier will be inserted here upon acceptance. 
amorphous and nanocrystalline magnetic materials are used for HFTs [16]. However, vendors provide only data sheets of core losses under sinusoidal voltage excitations, but the HFT generally operates under non-sinusoidal excitations. Therefore, extensive research is needed to characterize the advanced magnetic materials for their widespread uses.

In this paper, the factors affecting the design of HFTs and the state of the art design optimization processes are presented. The effects of each factor and its models have been reviewed. Moreover, the current research trend and future research directions are also discussed.

\section{State of ARt Magnetic Materials}

One of the key factors for the design of HFTs is the selection of appropriate core material. Both the efficiency and power density of an HFT depend on the core loss and the saturation flux density of the core materials. Till now, the silicon iron ( $\mathrm{SiFe}$ ) alloys are the best choice as the core material of low frequency laminated transformer. Some other conventional magnetic materials like nickel-iron $(\mathrm{NiFe})$ and cobalt iron $(\mathrm{CoFe})$ are also considered as core materials for low frequency transformer. However, for medium and high-frequency applications, due to higher core loss conventional magnetic materials are not suggested to use. In recent years, new soft magnetic materials like amorphous, nanocrystalline, and ferrites have been developed especially for high-frequency and/or high-speed electrical machine applications. The main problem of ferrites is the very low-saturation flux density, e.g. ferrites materials 3C85 and 3C93 present saturation flux densities of $0.45 \mathrm{~T}$ and $0.52 \mathrm{~T}$, respectively [17]. Therefore, machine designers are trying to develop HFT using amorphous and nanocrystalline magnetic materials.

\section{A. Amorphous Magnetic Materials}

Amorphous magnetic materials show very-low core loss because of their very thin foil structures, high-permeability and high-electrical resistivity [16]. The thickness and widths of commercially available amorphous ribbon are $15-35 \mu \mathrm{m}$ and 2.5-213 mm, respectively. Fig. 1 shows a photograph of the amorphous alloy ( $1 \mathrm{~kg}$ ribbon of $30 \mathrm{~mm}$ in width and $20 \mu \mathrm{m}$ in thickness). They also present lower manufacturing process cost compared to nanocrystalline magnetic materials. The first commercial amorphous material is the Metglas manufactured by Hitachi Metals Ltd., Japan in 1997 [32]. Metglas has mainly three types: iron $(\mathrm{Fe})$ based, cobalt $(\mathrm{Co})$ based and nickel $(\mathrm{Ni})$ based. Fe based Metglas amorphous materials are commonly used due to their availability. There are three commercial $\mathrm{Fe}$ based amorphous materials, which are 2605HB1, 2605SA1 and $2605 \mathrm{~S} 3 \mathrm{~A}$. The saturation flux density of $2605 \mathrm{HB} 1$ is the highest among them and it is $1.64 \mathrm{~T}$. The core losses of Meglas 2605SA1, 2605S3A and 2605HB1 materials are 10, 3 and 12 $\mathrm{W} / \mathrm{kg}$, respectively, at $0.2 \mathrm{~T}$ under a sinusoidal excitation of 10 $\mathrm{kHz}$ [32]-[34]. On the other hand, Co based amorphous materials, e.g. Metglas 2705M and 2714A show lower core losses with lower saturation flux density. Under $10 \mathrm{kHz}$ sinusoidal excitation of $0.2 \mathrm{~T}$, the iron loss is only $1.5 \mathrm{~W} / \mathrm{kg}$ for Metglas 2714A, whereas $10 \mathrm{~W} / \mathrm{kg}$ for Metglas 2605SA1 [32]-
[34]. Due to very expensive cobalt content, Co based materials are more expensive than the $\mathrm{Fe}$ based materials. $\mathrm{Ni}$ based amorphous material such as Metglas $2826 \mathrm{MB}$ is also used for high-frequency applications. Core loss of Metglas 2714A is the lowest among the Metglas amorphous materials. However, its lower saturation flux density and high price hinder its applications. Recently, Vacuumschelze GmbH \& Co. KG has developed new Co based amorphous materials such as VITROVAC $6025 \mathrm{~F}$, VITROVAC $6030 \mathrm{~F}$ and VITROVAC $6155 \mathrm{~F}$ [35]. Their core losses at $0.3 \mathrm{~T}$ and $100 \mathrm{kHz}$ are comparable with that of Meglas 2714A, as shown in Table I.

TABLE I

COMPARISON OF DIFFERENT AMORPHOUS MATERIALS [32]-[35]

\begin{tabular}{lllll}
\hline \hline $\begin{array}{l}\text { Amorphous } \\
\text { magnetic materials }\end{array}$ & $\begin{array}{l}\mathrm{B}_{\text {sat }} \\
(\mathrm{T})\end{array}$ & $\begin{array}{l}\text { Core loss }(\mathrm{W} / \mathrm{kg}) \\
0.3 \mathrm{~T} \& 100 \mathrm{kHz}\end{array}$ & $\begin{array}{l}\text { Resistivity } \\
(\mu \Omega . \mathrm{cm})\end{array}$ & $\begin{array}{l}\text { Density } \\
\left(\mathrm{g} / \mathrm{cm}^{3}\right)\end{array}$ \\
\hline VITROVAC 6025 F & 0.40 & 100 & 1.40 & 7.8 \\
VITROVAC 6030 F & 0.70 & 110 & 1.30 & 7.75 \\
VITROVAC 6155 F & 0.90 & 130 & 1.10 & 7.90 \\
Metglas 2705M & 0.77 & 260 & 1.42 & 7.59 \\
Metglas 2714A & 0.57 & 120 & 1.38 & 7.90 \\
Metglas 2605S3A & 1.41 & 300 & 1.38 & 7.29 \\
Metglas 2605SA1 & 1.56 & 1400 & 1.30 & 7.18 \\
Metglas 2826MB & 0.88 & 1360 & 1.38 & 7.90 \\
\hline
\end{tabular}

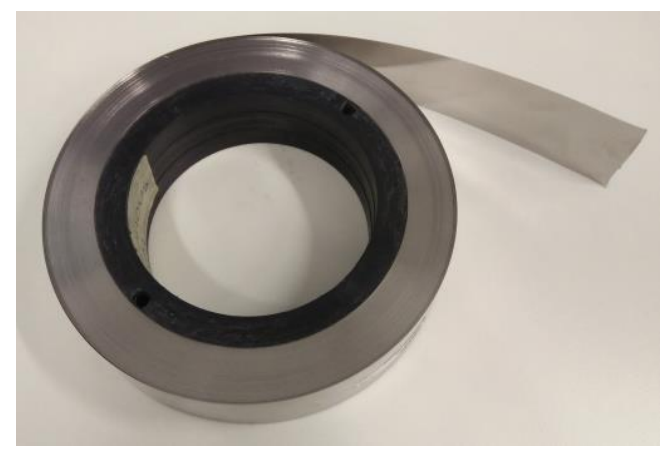

Fig. 1. A photograph of the amorphous alloy (1 $\mathrm{kg}$ ribbon of $30 \mathrm{~mm}$ in width and $20 \mu \mathrm{m}$ in thickness).

\section{B. Nanocrystalline Magnetic Materials}

Nanocrystalline magnetic material has received a great attention due to its lower core loss than amorphous magnetic materials. VITROPERM and FINEMET are commonly used nanocrystalline magnetic materials which have core losses of 1.5 and $5 \mathrm{~W} / \mathrm{kg}$, respectively under $10 \mathrm{kHz}$ sinusoidal excitation of $0.3 \mathrm{~T}$, whereas Metglas 2605S3A features $7 \mathrm{~W} / \mathrm{kg}$. However, the fabrication process of nanocrystalline is quite difficult and expensive. Recently, $\mathrm{Fe}_{81.8} \mathrm{Cu}_{1.0} \mathrm{Mo}_{0.2} \quad \mathrm{Si}_{4} \mathrm{~B}_{14}$ and $\mathrm{Cu}_{\text {bal. }} \mathrm{Cu}_{1} \mathrm{Mo}_{0.2} \mathrm{Si}_{4} \mathrm{~B}_{14}$ nanocrystalline alloy ribbons have been developed which show higher saturation flux density than the conventional nanocrystalline and amorphous materials [36], [37]. The core losses at $1 \mathrm{~T}$ and $1 \mathrm{kHz}\left(\mathrm{P}_{10 / 1 \mathrm{k}}\right)$, and at $0.2 \mathrm{~T}$ and $10 \mathrm{kHz}\left(\mathrm{P}_{2 / 10 \mathrm{k}}\right)$ for different soft magnetic materials are shown in Table II. The lower core loss is obtained for FINMET nanocrstalline alloy but its lower saturation induction makes obstacle for high power density applications. On the other hand, the $\mathrm{Fe}_{81.8} \mathrm{Cu}_{1.0} \mathrm{Mo}_{0.2} \mathrm{Si}_{4} \mathrm{~B}_{14}$ nanocrystalline alloy not only exhibits higher saturation flux density but also exhibits lower core loss. 
TABLE II

COMPARISON OF DIFFERENT MAGNETIC MATERIALS [36]

\begin{tabular}{llll}
\hline \hline $\begin{array}{l}\text { Different soft } \\
\text { materials }\end{array}$ & $\begin{array}{l}\mathrm{B}_{\text {sat }} \\
(\mathrm{T})\end{array}$ & $\begin{array}{l}\left(\mathrm{P}_{10 / 1 \mathrm{k}}\right) \\
(\mathrm{W} / \mathrm{kg})\end{array}$ & $\begin{array}{l}\left(\mathrm{P}_{2 / 10 \mathrm{k}}\right) \\
(\mathrm{W} / \mathrm{kg})\end{array}$ \\
\hline $\mathrm{Fe}_{81.8} \mathrm{Cu}_{1.0} \mathrm{Mo}_{0.2} \mathrm{Si}_{4} \mathrm{~B}_{14}$ & 1.75 & 5 & 8 \\
Metglas 2605HB1 alloy & 1.64 & 4.4 & 12 \\
$6.5 \mathrm{wt} \% \mathrm{Si}$-steel & 1.80 & 18.1 & 30 \\
FINEMET (FT3) alloy & 1.25 & -- & $<2$ \\
\hline
\end{tabular}

\section{CORE-LOSS MODELING OF HFT}

In HFT's design, one of the main challenges is to predict the core loss accurately. The core loss increases with operating frequency and flux density. Several models in the literatures have been proposed to predict the core loss in the last few decades. These models can be grouped into two approaches: loss separation model and Steinmetz based model. In the first method, the core loss has three parts: hysteresis loss, eddy current loss and the anomalous or excess loss [38]. Authors of [39] proposed a loss separation model for sinusoidal excitation which is given by

$$
P=K_{h} B_{m}^{a B_{m}^{2}+b B_{m}+c} f+K_{e} B_{m}^{2} f^{2}+8.76 K_{e x c} B_{m}^{1.5} f^{1.5}
$$

where $f$ is the frequency, $B_{m}$ is the maximum flux density and $K_{h}, K_{e}, K_{e x c}, a, b$, and $c$ are the constant coefficients. The measurements of these coefficients are extensive which makes the method impractical for designers. In contrast, the Steinmetz based empirical methods are more practical and straightforward to predict the core losses. The method was first proposed by Chas Proteus Steinmetz [40]. In this method, a generalized equation for core loss in magnetic material is proposed which is given by [40],

$$
P=k f^{\alpha} B_{m}^{\beta}
$$

where $k, \alpha$ and $\beta$ are the Steinmetz parameters. The main downside of original Steinmetz equation (OSE) is that the equation is valid for only sinusoidal excitation. However, in HFT topologies, the excitation voltage is non-sinusoidal. Moreover, the nonlinear relationship between excitation and flux density makes it impossible to apply Fourier series approach to evaluate the core loss. Therefore, many models have been proposed to predict the core loss for non-sinusoidal excitation. The OSE was first modified by authors of [41] where the core loss is also related to the rate of the change of magnetic flux density which is given by

$$
P=k f_{e q}^{\alpha-1} B_{m}^{\beta} f_{r}
$$

where $f_{r}$ is the fundamental frequency and $f_{e q}$ is given by

$$
f_{e q}=\frac{2}{\Delta \beta^{2} \pi^{2}} \int_{0}^{T}\left(\frac{d B}{d t}\right)^{2} d t
$$

In [42], it is shown that core loss not only depends on the rate of the change of the flux density but also depends on its instantaneous value. The authors proposed the following generalized Steinmetz equation (GSE) for prediction of core loss [42]

$$
P=\frac{1}{T} \int_{0}^{T} k_{1}\left|\frac{d B(t)}{d t}\right|^{\alpha}|B(t)|^{\beta-\alpha} d t
$$

$$
\text { where } k_{1}=\frac{k}{(2 \pi)^{\alpha-1} \int_{0}^{2 \pi}|\cos \theta|^{\alpha}|\sin \theta|^{\beta-\alpha} d \theta} \text {. }
$$

In [43], authors showed that the time history of magnetic material also affects the core loss. For that, the instantaneous value of flux density is substituted by the peak to peak value and the equation of the loss is given by [43]

$$
P=\frac{1}{T} \int_{0}^{T} k_{1}\left|\frac{d B(t)}{d t}\right|^{\alpha}|\Delta B|^{\beta-\alpha} d t
$$

$$
\text { where } k_{1}=\frac{k}{(2 \pi)^{\alpha-1} \int_{0}^{2 \pi}|\cos \theta|^{\alpha} 2^{\beta-\alpha} d \theta} \text {. }
$$

The author of [44] proposed another modified Steinmetz equation known as natural Steinmetz equation (NSE) to predict the core loss. The equation of NSE method is as follows [44]

$$
P=\left(\frac{\Delta B}{2}\right)^{\beta-\alpha} \frac{K_{N}}{T} \int_{0}^{T}\left|\frac{d B(t)}{d t}\right|^{\alpha} d t
$$

$$
\text { where } k_{N}=\frac{k}{(2 \pi)^{\alpha-1} \int_{0}^{2 \pi}|\cos \theta|^{\alpha} d \theta} \text {. }
$$

The simplest way to predict the core loss for non-sinusoidal excitation with slight modification of Steinmetz equation was proposed in [45], known as waveform coefficient Steinmetz equation (WCSE). A factor $\left(K_{\mathrm{FWC}}\right)$ which is the ratio of areas of the non-sinusoidal and sinusoidal waveforms is just multiplied by the original Steinmetz equation. The equation of the waveform coefficient Steinmetz equation for any arbitrary excitation voltage is given by [45]

$$
P=K_{F W C} k f^{\alpha} B_{m}^{\beta} .
$$

A loss occurs in the zero voltage time due to the relaxation process. This loss is not considered in the abovementioned methods. In [46], a new empirical method was proposed to predict the core loss considering the relaxation process in zero voltage interval. The total core-loss can be evaluated by [46]

$$
P=\frac{1}{T} \int_{0}^{T} k_{1}\left|\frac{d B(t)}{d t}\right|^{\alpha}|\Delta B|^{\beta-\alpha} d t+\sum_{l=1}^{n} Q_{r l} P_{r l}
$$

where $P_{r l}$ and $Q_{r l}$ are given by

$$
\begin{aligned}
& P_{r l}=\frac{1}{T} k_{r 1}\left|\frac{d B\left(t_{-}\right)}{d t}\right|^{\alpha_{r}}|\Delta B|^{\beta_{r}}\left(1-e^{-\frac{t_{1}}{\tau}}\right) \\
& Q_{r l}=e^{-q_{r}\left|\frac{d B\left(t_{+}\right) / d t}{d B\left(t_{-}\right) / d t}\right|}
\end{aligned}
$$

where all constantans are material parameters. Recently, the authors in [47] and [48] calculated Steinmetz parameters to model core loss of HFT under square-wave excitation and characterize amorphous core under square-wave excitation. They also compared the core loss of amorphous alloy for both sinusoidal and non-sinusoidal excitations. Fig. 2 shows the 
comparison of core loss of Metglas 2605SA1 for square and sinusoidal excitations.

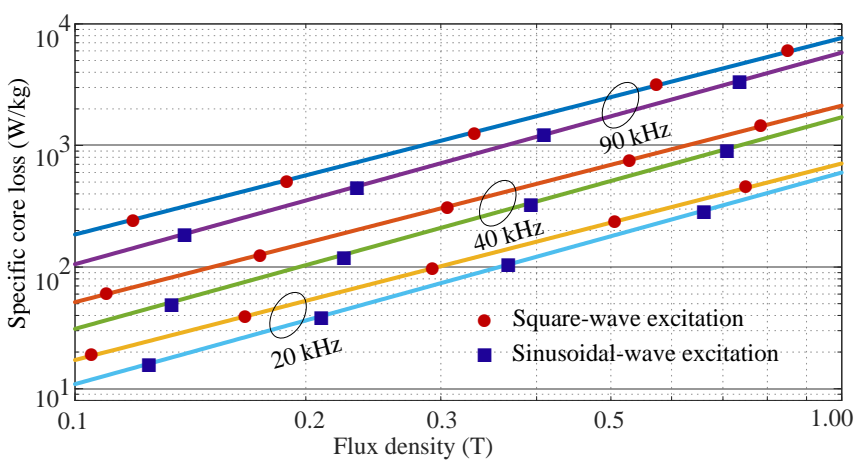

Fig. 2. Comparison of core loss of Metglas 2605SA1 under square and sinusoidal excitations.

\section{EXPERIMENTAL TECHNIQUES FOR CORE LOSS MEASUREMENT}

Magnetic core loss is an important concern in the area of HFT design. Since the core loss depends on the frequency, the increase of operating frequency affects the efficiency and heat dissipation. Consequently, at high frequency range the accurate core loss measurement becomes significant. There are many core loss measurement techniques reported in the literature which can be classified into mainly two groups: thermal or calorimeter techniques and electrical techniques [49].

\section{A. Thermal or Calorimeter Techniques}

In the thermal method, different types of coolants, e.g. water and air are used to absorb heat produced due to core loss. As a result, temperature of the coolant increases which indicates the power dissipation. The power loss can be obtained from the temperature difference and flow rate of coolant. In [19], the authors proposed a calorimeter to measure the core loss. A core under test (CUT) is placed inside the vessel where FC-40 is used as coolant which flows at a constant rate with a pump and control valve, as shown in Fig. 3. Under steady state condition, the final temperature difference is measured. Finally, the total power dissipated is calculated by [19]

$$
P=\rho A V C_{s} \Delta T
$$

where $\rho$ is the density of the coolant, $V$ the flow rate of the coolant, $C_{s}$ the specific heat of fluid and $A$ the cross section of coolant path. To reduce the loss due to the convection and convection and radiation, the authors of [50] proposed an improved calorimeter technique to measure the core loss where the CUT is placed inside a vacuum chamber and the test is done under isothermal conditions. However, the thermal methods have some common disadvantages, e.g. time consuming, difficulty of separating winding loss from core loss, higher error in case of low core loss and difficulty of making test platform [49].

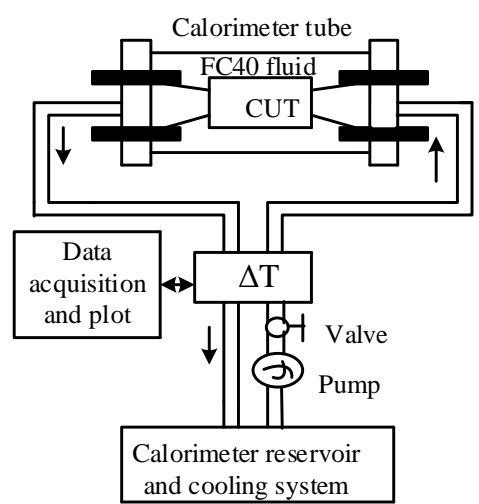

Fig. 3. The block diagram of core loss measurement using calorimeter [19].

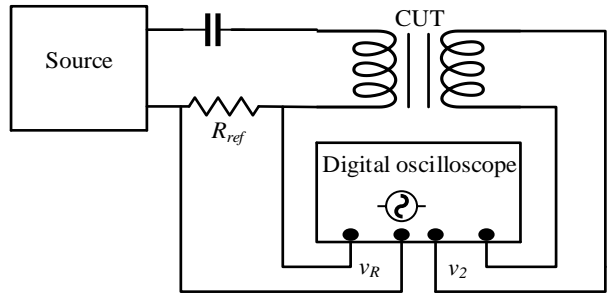

Fig. 4. Schematic diagram of core loss measurement using oscilloscope [49].

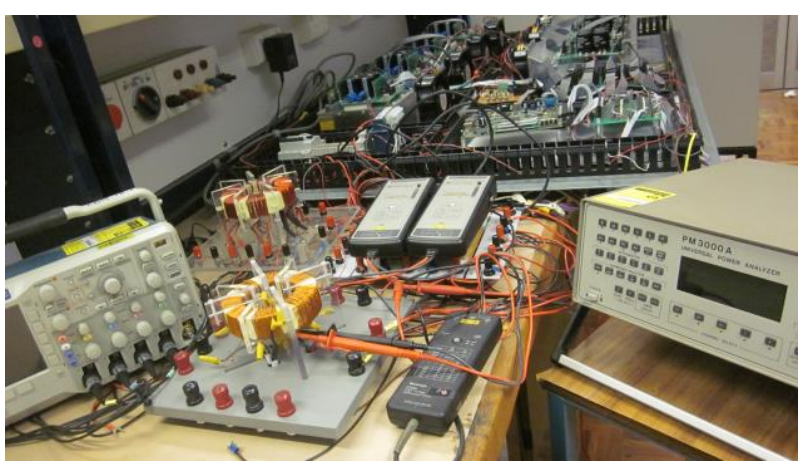

Fig. 5. A photograph of the test platform to measure specific core loss of highfrequency transformer [51].

\section{B. Electrical Techniques}

The disadvantages of thermal techniques can be eliminated by the electrical techniques. The electrical techniques are classified into two groups: impedance technique and wattmeter technique. In impedance techniques, the core loss is considered as an equivalent loss resistance, which may be associated with parallel or series with the magnetizing inductance. The Maxwell-Wien Bridge and network analyzer are generally utilized in this approach to measure the core loss. The wattmeter technique is the mostly used technique to measure core loss of magnetic materials where two windings are wound on a core used for experiment as shown in Fig. 4. Instead of traditional wattmeter, oscilloscopes are commonly exploited to measure core loss due to non-sinusoidal excitation [52]. A photograph to measure core loss of HFT is shown in Fig. 5. The excitation current and secondary open circuit voltage are used for calculation of core loss by the following equation [52]:

$$
P=\frac{1}{T} \int i_{p}(t) v_{s}(t) d t
$$


where $i_{p}$ and $v_{s}$ are the primary current and secondary voltage, respectively. In this method, dc bias in magnetization current is not included during the core loss measurement set up. The experimental set up for core loss measurement with dc bias is shown in Fig. 6. A digital oscilloscope is exploited to sample the excitation current and voltage. Finally, the core loss is calculated from sampled data by using the following equation [53]:

$$
P=\frac{N_{p}}{N_{s}} \frac{1}{N} \sum_{i=1}^{N} v_{s} \frac{v_{R}}{R_{r e f}}
$$

where $N_{p}$ and $N_{s}$ are the number of turns in primary and secondary windings respectively, and $R_{r e f}$ the resistance of current sensor and $v_{R}$ the voltage across the current sensor resistor and $N$ the number of sample per period. To reduce the effect of phase discrepancy which exists in abovementioned methods, a resonant capacitor or ideal inductor is connected in series with excitation winding as shown in Fig. 7 [20], [54], [55]. In these approaches, the sum of voltage of secondary winding and capacitor or inductor voltage is closely equal to the voltage due to core loss. Consequently, the effect of phase discrepancy will reduce. The value of resonant capacitor $\left(C_{r}\right)$ is given by [20]

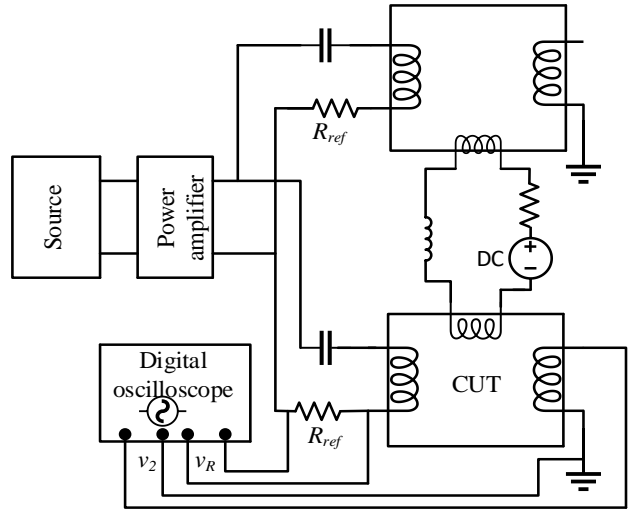

Fig. 6. Schematic diagram of core loss measurement with dc bias [53].

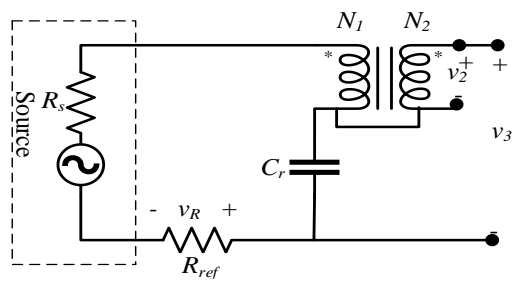

Fig. 7. Core loss measurement using capacitive cancellation [20].

$$
C_{r}=\frac{N_{p}}{N_{s}(2 \pi f)^{2} L_{m}}
$$

where $L_{m}$ is the magnetizing inductance of the winding. In [56], partial inductive and capacitive cancellation methods are also proposed to decrease the effect of phase discrepancy. In partial cancellation methods, a fraction of capacitive or inductive voltages is employed in capacitive and inductive cancellation instead of full capacitive or inductive voltages [56].

\section{WINDING AC RESISTANCE MODELING}

Winding loss or copper loss depends on the resistance of the winding wires and affects the performance of a transformer. The copper loss increases due to the increase of ac resistance $\left(R_{a c}\right)$ of the wires with the increase of excitation frequency, as shown in Fig. 8.

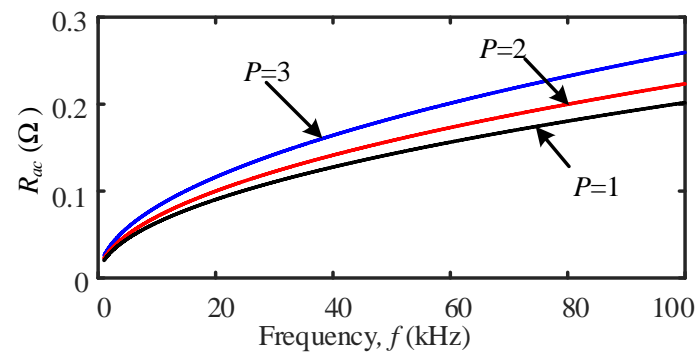

Fig. 8. $R_{a c}$ for different layers with frequency.

Due to the skin and proximity effect, the distribution of current across the cross section of a conductor is not uniform. This non-uniform current distribution makes a difference in the values of ac resistance from dc value. One dimensional Maxwell's equation is first exploited to derive the expression of leakage impedance [23]. The Dowell's expression of leakage resistance is given by (16) [23]

$$
R_{a c}=\frac{p \rho N_{l}^{2} l_{t}}{\eta b h}\left\{M^{\prime}+\frac{\left(p^{2}-1\right) D^{\prime}}{3}\right\}
$$

where $b$ is the winding breadth, $\rho$ resistivity, $\eta$ porosity factor, $p$ the number of layers, $N_{l}$ number of turns per layer, $l_{t}$ mean turn length, $h$ height of conductor, and $M^{\prime}$ and $D^{\prime}$ are the real parts of $M$ and $D$, respectively, which are as follows [23]:

$M=\alpha h \operatorname{coth}(\alpha h), D=2 \alpha h \tanh (\alpha h / 2), \alpha=\sqrt{\frac{j \omega \eta \mu}{\rho}}$

where $\omega$ is angular frequency, and $\mu$ is the permeability of conductor. Due to cylindrical windings in most transformers, the expression of ac resistance is derived as shown in (17) by using the cylindrical coordinates instead of rectangular coordinates which considers the curvature of wound wire [57], [58].

$$
R_{a c}=R_{d c} \Delta\left[\frac{\sinh 2 \Delta+\sin 2 \Delta}{\cosh 2 \Delta-\cos 2 \Delta}+\frac{2\left(p^{2}-1\right)}{3} \frac{\sinh \Delta-\sin \Delta}{\cosh \Delta+\cos \Delta}\right]
$$

where $R_{d c}$ is the dc resistance of the winding, $\Delta$ the ratio of thickness of conductor and skin depth, and $p$ is the number of layers. The aforementioned expressions of ac resistance have some shortcomings, e.g. the effect of magnetizing current is not considered, round conductor is replaced by square conductor and the skin depth is considered as a function of porosity factor. These shortcomings can be eliminated by more exact analytical methods in [59] and [60]. The ac resistance of a solid round conductor is given by [59], [60]

$$
\begin{array}{r}
R_{a c}=R_{d c} \frac{\gamma}{2}\left[\frac{b e r \gamma b e i^{\prime} \gamma-b e i \gamma b e r^{\prime} \gamma}{b e r^{\prime 2} \gamma+b e i^{\prime 2} \gamma}\right. \\
\left.-2 \pi(2 p-1)^{2} \frac{\text { ber }_{2} \gamma b e r^{\prime} \gamma+b e i_{2} \gamma b e i^{\prime} \gamma}{b e r^{2} \gamma+b e i^{2} \gamma}\right]
\end{array}
$$


where $\gamma=h /(\sqrt{2} \delta)$ and $h$ is the thickness of conductor, $\delta$ the skin depth. In the abovementioned methods, it is assumed that the magnetic field across the cross section of the conductor is uniform. In [61], this consideration is eliminated and a new expression of ac resistance is developed which is given below

$$
\begin{aligned}
& R_{a c}=R_{d c} \frac{\gamma}{2}\left[\frac{b e r \gamma b e i^{\prime} \gamma-b e i \gamma b e r^{\prime} \gamma}{b e r^{\prime 2} \gamma+b e i^{\prime 2} \gamma}-\right. \\
&\left.2 \pi \eta^{2}\left(4 \frac{p^{2}-1}{3}+1\right) \frac{\operatorname{ber}_{2} \gamma b \operatorname{cr}^{\prime} \gamma-b e i_{2} \gamma b e i^{\prime} \gamma}{b e r^{2} \gamma+b e i^{2} \gamma}\right]
\end{aligned}
$$

Equations (16)-(19) are derived for only sinusoidal excitation. For non-sinusoidal excitation, Fourier series expansion can be utilized for determining the ac resistance. The main problem of the approach is determination of Fourier coefficients. The authors of [57] proposed a method to derive the expression of ac resistance without calculating the Fourier coefficients. The expression for leakage resistance is given by [57]

$$
R_{a c}=1+\frac{\left(5 p^{2}-1\right)}{45} \Delta^{4}\left(\frac{I_{r m s}^{\prime}}{\omega I_{r m s}}\right)^{2}
$$

where $I_{r m s}$ is the root mean square value of current and $I_{r m s}^{\prime}$ is the derivative of $I_{r m s}$.

\section{LEAKAGE INDUCTANCE MODELING}

Leakage inductance is another parasitic element of HFT which also affects the performance of HFT. Just like leakage resistance, the leakage inductance in high frequency differs from its static value and changes with the frequency due to eddy current and proximity effects, as shown in Fig. 9. The analytical expressing of leakage inductance was first obtained from the Dowell's expression which is as follows [23]:

$$
L=\frac{\mu_{0} p^{3} N_{l}^{2} h l_{t}}{3 b}\left[\frac{3 M^{\prime \prime}+\left(p^{2}-1\right) D^{\prime \prime}}{p^{2}\left|\alpha^{2} h^{2}\right|}\right]
$$

where $M^{\prime \prime}$ and $D^{\prime \prime}$ are the imaginary parts of $M$ and $D$ respectively. In [62] and [25], the total leakage inductance is obtained from the stored energy. The simplified equation of the total leakage inductance with considering one turn per layer is obtained from the sum of all stored magnetic energies which is given below [62]

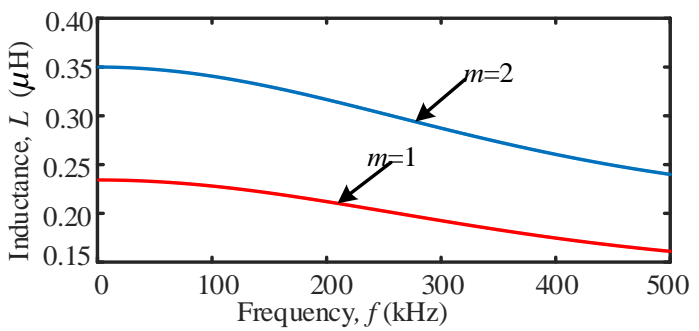

Fig. 9. Leakage inductance with frequency for turn ratio 1 and 2.

$$
L_{l k}=\frac{\mu_{0} l_{t} n_{p}}{6 h_{w}}\left[\frac{n_{p}^{2}\left(k_{1}+2 k_{2}\right)(m+1)}{\gamma \sinh ^{2}\left(\not t_{p}\right)}+\frac{\left(k_{1}-4 k_{2}\right)(m+1)}{2 n \gamma \sinh ^{2}\left(\mu_{p}\right)}\right.
$$

$$
\left.+\left\{2 n_{p}^{2}+2 m n_{p}^{2}+\frac{1}{m}+1\right\} t_{\text {iso }}\right]
$$

where, $k_{1}=\sinh \left(2 \gamma t_{p}\right)-2 \gamma t_{p}, k_{2}=\gamma t_{p} \cosh \left(\gamma t_{p}\right)-\sinh \left(\gamma t_{p}\right), n_{p}$ is the number of layers in the primary winding, $m$ the turn ratio, $t_{p}$ the primary conductor thickness, $t_{i s o}$ insulation thickness and $h_{w}$ core window height. In [63], the effect of winding position on core is investigated. The authors showed that the value of leakage inductance also changes with the winding position and the air gap between two windings. In addition, the wide range of leakage inductance as a part of resonant converter can also be obtained by changing the position of windings.

\section{StRay CaPacitance Modeling}

The performance of the converter is affected by the stray or parasitic capacitance of HFT. Due to the stray capacitance, the system may develop resonant frequency which creates electromagnetic interference and unwanted oscillation in primary and secondary currents of HFT. The parasitic capacitance of HFT mainly comprises of turn to turn capacitance, single layer capacitance, layer to layer capacitance and inter-windings capacitance [64]. Here, inter-windings capacitance is neglected.

\section{A. Turn to Turn Capacitance}

Turn to turn capacitance is the basic element of parasitic or stray capacitance. The elementary capacitance between two conductors consists of series connected elementary insulation capacitance and air capacitance. The main challenge of determination of capacitance is to calculate the effective distance of electric field path which is shown in Fig. 10. Due to lower insulation distance, in [64]-[66], the electric field line in the insulation is considered as radial, and the radial distance can easily be measured from their diameters. Therefore, the insulation capacitance can be calculated by [66]

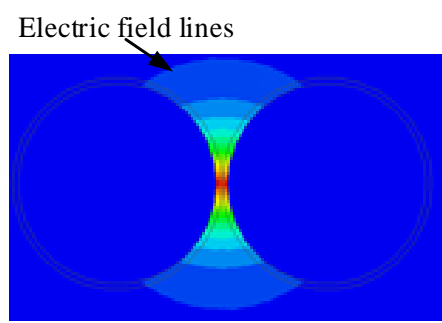

(a)

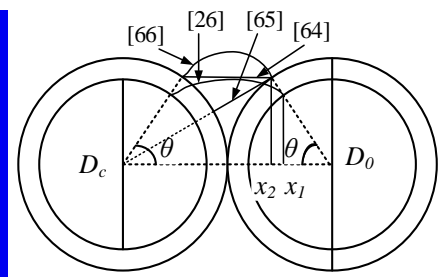

(b)
Fig. 10. (a) Distribution of electric field, and (b) different proposed path of electric field lines [26], [64]-[66].

$$
d C_{t t c}(\theta)=\frac{\varepsilon_{s} l_{t}}{\ln \frac{D_{0}}{D_{c}}} d \theta
$$

where $d C_{t t c}$ is the elementary insulation capacitance, $\varepsilon_{s}$ the permittivity of insulation, $l_{t}$ the length per turn, $\theta$ the deflection angle, $D_{0}$ and $D_{c}$ the diameters of solid wire including and excluding insulation layer, respectively. In [26], however, the distance is considered as a curve of a parabola. The equations of parabola and insulation capacitance are as follows [26]: 


$$
y=\frac{2 x^{2} \sin \theta\left(D_{c}-D_{0}\right)}{\left(D_{0}-D_{c} \cos \theta\right)^{2}}+\frac{D_{0}}{2} \sin \theta
$$

and

$$
d C_{t t c}=\frac{\varepsilon_{s} D_{0}}{2 \int_{x_{1}}^{x_{2}} \sqrt{1+\left(\frac{d y}{d x}\right)^{2}} d x} d \theta
$$

On the other hand, to determine the effective distance of electric path in the air, the path is considered by many approaches, e.g. parallel to the line between two centers of conductors, radial until it touches the surface of other conductor, curve of a circle or parabola as shown in Fig. 10 [26], [64]-[66]. The equations of different effective distances in the air using aforementioned ways are given by (26)-(29) [64]-[66]. Finally, the air capacitance and total capacitance can be calculated by (30) and (31) respectively [26]

$$
\begin{aligned}
& x(\theta)=D_{0}(1-\cos \theta) \\
& x(\theta)=D_{0}\left(\cos \theta \pm \sqrt{\cos ^{2} \theta-\frac{3}{4}}-\frac{1}{2}\right) \\
& x(\theta)=\theta \times D_{0} \times \tan (\theta / 2) \\
& x(\theta)=\int_{-x_{1}}^{x_{1}} \sqrt{1+\left(\frac{d y}{d x}\right)^{2}} d x \\
& d C_{t t g}(\theta)=\frac{\varepsilon_{0} \frac{l_{t} D_{0}}{2}}{x(\theta)} d \theta \\
& d C_{t t e q}(\theta)=\int_{\theta_{1}}^{\theta_{2}} \frac{1}{d C_{t t c}}+\frac{1}{d C_{t t g}}
\end{aligned}
$$

where $d C_{t t g}$ and $d C_{t t e q}$ are the elementary air capacitance and total equivalent capacitance, and $\varepsilon_{0}$ is the permittivity of air.

\section{B. Single Layer Capacitance}

There are many turns in a layer. If the capacitance between turn and shield or core is neglected, the single layer capacitance is equal to $C_{t t} /(n-1)$, where $n$ is number of turn of a single layer. The capacitance between turn and shield is considered as shown in Fig. 11 and the capacitance between two terminals of the winding is given by [64], [65]

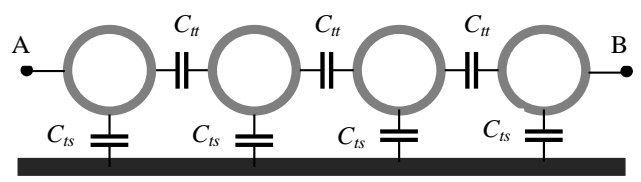

Fig. 11. Equivalent network of turn to turn and turn to shield or core [65].

$$
C_{A B}(n)=\frac{C_{t t}}{2+\frac{C_{t t}}{C_{A B}(n-2)}}+C_{t t} .
$$

The shield capacitance $\left(C_{t s}\right)$ is considered as double of turn to turn capacitance for simplicity. The effect of non-adjacent turns is also neglected for simplicity.

\section{Layer to Layer Capacitance}

Capacitance also exists between two layers due to voltage difference between them. The capacitance between two layers can be obtained by [65]

$$
C_{l l}=\frac{n(n+1)(2 n+1)}{6 n^{2}} l C_{t t}
$$

where $C_{l l}$ is the layer to layer capacitance and $l$ is the average length of a turn. For higher turn ratio, multi-layer winding exists. So the capacitance for multilayer winding is also required to determine. The capacitance for multilayer winding can be calculated using layer to layer capacitance, whose equation is as follows [65]

$$
C_{p}=C_{l l}(z-1)(2 / z)^{2}
$$

where $z$ is the number of layers of a winding. Equation (34) can be utilized to calculate the stray capacitance of primary and secondary windings.

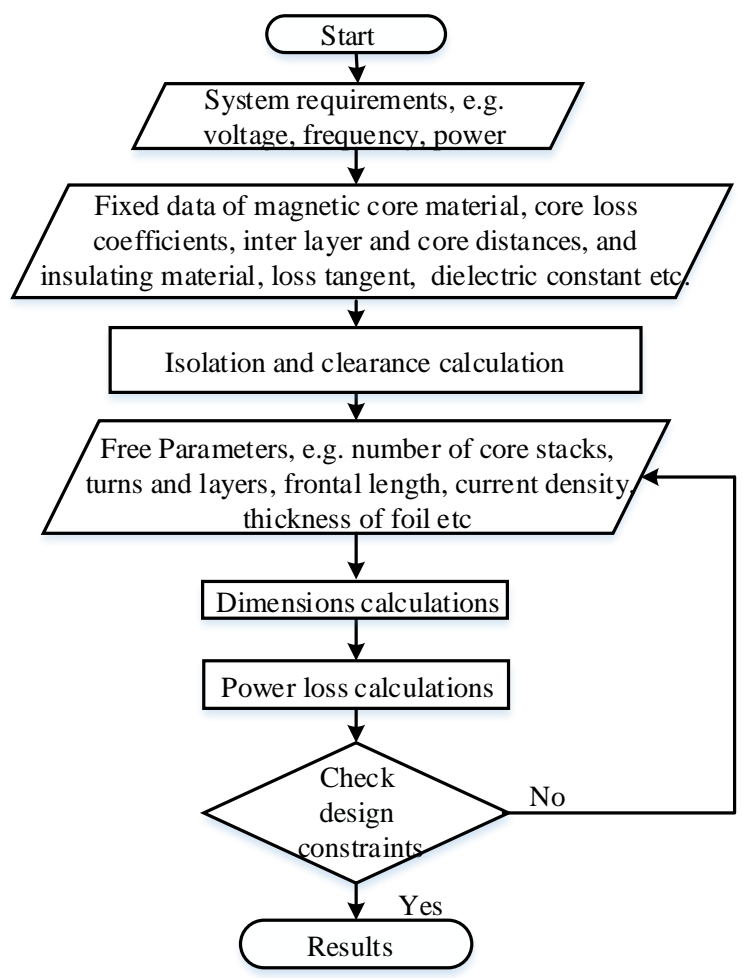

Fig. 12. The flow chart of an optimal design of HFT [67].

\section{DESIGN OPTIMIZATION}

It is important to optimize the design of an HFT to achieve high power density and efficiency with all requirements, e.g. lower leakage inductance, proper thermal management and isolation. Most of the traditional optimal techniques are based on the iterative approaches where a few parameters such as the efficiency and maximum temperature limit, are focused by optimizing variable parameters. Fig. 12 shows a general optimization technique. 


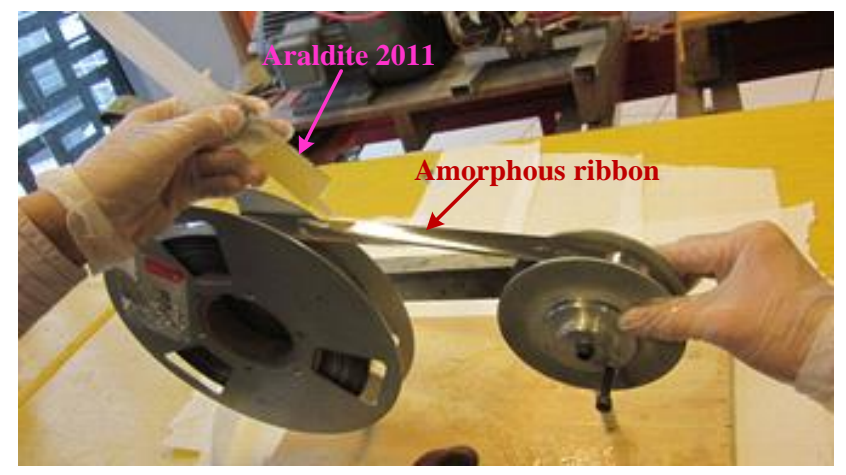

Fig. 13. A photograph of the HFT core development process with amorphous ribbon [71].

In the above optimization technique, core materials, core stacks, cross section, layer number, turn ratio, conductor thickness and current density are normally fixed variables [67]. Then different design parameters like geometrical parameters, core losses, winding losses, temperature and isolation distance are solved for all set of variable in specified range. Finally, the set of parameters which shows high power density and efficiency in specific temperature are selected for optimal design.

The authors of [68]-[70] proposed computer based heuristic optimal algorithms, e.g. artificial neural network (ANN), multiobjective genetic algorithm (MOGA) and Numeric Pareto geometry optimization process to design high frequency transformer which requires minimum number of parameters compared to the aforementioned methods. Based on design parameters as obtained from optimization process an optimal core can be designed in the laboratory with the commercial advanced magnetic ribbon. The authors of [71] and [72] introduced the concept to develop HFT core with $\mu \mathrm{m}$ thick magnetic ribbon. Fig. 13 shows a photograph of the HFT core development process with amorphous ribbon.

\section{COUPlED FIELD-CIRCUIT APPROACH}

For flux distribution, stability, reliability and transient analysis of HFT, coupled field-circuit approach can be used. The coupled field-circuit approach has two parts: field part and circuit part [73]. The field and circuit parts are generally analyzed by finite-element method (FEM) and nodal analysis method respectively. In [74], the coupled field circuit approach was utilized to analyze the HFT. The obtained mathematical model is as follows [74]:

$$
\left[\begin{array}{cc}
\mathbf{T}_{u} & 0 \\
2 \lambda_{e f} \mathbf{C}_{r m}^{T} & L_{l}
\end{array}\right]\left[\begin{array}{c}
\frac{\partial \mathbf{A}}{\partial t} \\
\frac{\partial i}{\partial t}
\end{array}\right]+\left[\begin{array}{cc}
\mathbf{S} & -\mathbf{C}_{r m} \\
0 & R
\end{array}\right]\left[\begin{array}{c}
\mathbf{A} \\
i
\end{array}\right]=\left[\begin{array}{c}
\mathbf{F} \\
u
\end{array}\right]
$$

where $L_{l}$ is the diagonal matrix of leakage inductances, $R$ the diagonal matrix of winding resistances, $u$ the vector of input and output voltages, $i$ the vector of input and output currents, $\mathbf{C}_{r m}$ the correlation matrix, $\lambda_{\text {ef }}$ the length of field along the axial direction, $\mathbf{T}_{u}$ the matrix related to area and conductivity, $\mathbf{F}$ the vector of field sources, $\mathbf{A}$ the vector potential and $\mathbf{S}$ the function of the curl of A. However, FEM based softwares such as ANSYS and COMSOL are currently being popular as a design and analysis tool of HFT due to more user friendly than coupled field-circuit approach. Using ANSYS flux distribution of a toroidal core based HFT is shown in Fig. 14.

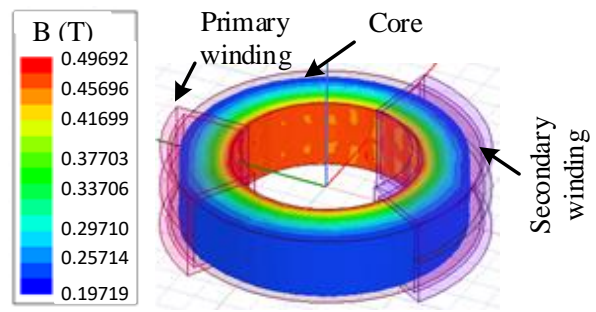

Fig. 14. Magnetic flux density at time, $t=0.00012 \mathrm{~s}$ of a toroidal core based HFT operating at $25 \mathrm{kHz}$.

\section{Thermal Management Modeling}

The thermal analysis is one of the major constituents in the HFT design process. Since the whole power losses are dissipated as heat, the temperature of the transformer rises which affects the performance. In case of low power HFT's design, thermal management is often avoided due to low amount of losses. For medium and higher density, however, the analysis is important to achieve higher performance and safe operation. Authors of [71] analyzed heat distribution in an HFT with a thermal imaging camera. Fig. 15 shows a thermal image of an HFT with amorphous magnetic core and Litz wire windings under operation. However, the amount of increased temperature due to loss is obtained by the following equation [75]:

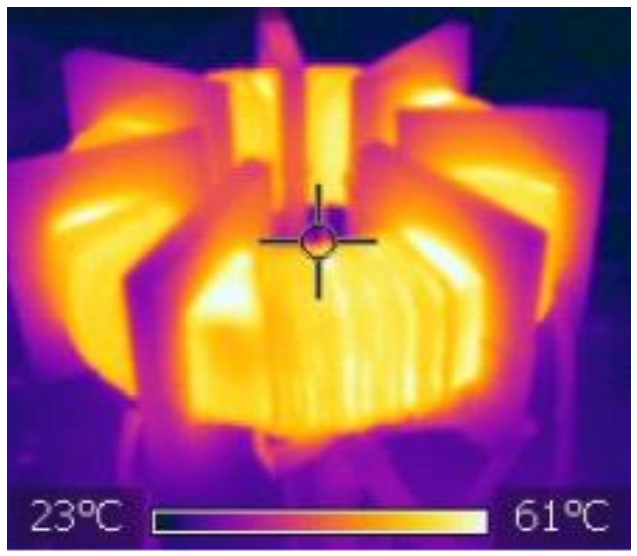

Fig. 15. Temperature distribution in an HFT with amorphous magnetic core and Litz wire windings [72].

$$
\Delta T=P_{\text {total }} R_{\text {th }}
$$

where $\Delta T$ is the temperature rise, $R_{t h}$ the thermal resistance and $P_{\text {total }}$ the transformer total loss. Compared to the theoretical methods, empirical models are simple and easy to incorporate in the HFT design. Moreover, empirical models provide almost the same accuracy as the theoretical models. In [75], the authors used the following empirical expression to predict $R_{t h}$,

$$
R_{t h}=\frac{0.0457}{\sqrt{V_{c}}}
$$

where $V_{c}$ is the volume of the core. Since one of the HFT design constraints is the thermal limit, the transformer must operate below the maximum permissible temperature rise, i.e. $P_{\text {total }} R_{t h}$ 
$\leq \Delta T_{\max }$, where $\Delta T_{\max }$ is the maximum permissible temperature rise.

In the conventional low-frequency transformer, transformer oil is used as insulation and cooling medium. However, due to environment and safety reasons, transformer oil free HFT design is preferred. To achieve efficient cooling concept for high power HFT, heat from core is extracted from two ways [10], [27], [28]. One way is the heat extraction by heat sinks done from the lower and upper surfaces of the core where heat also comes from the limbs due to thermal conduction [27]. Forced air or water cooled heat sinks are generally exploited as heat sinks. Another way is the direct heat extraction from limbs where the water cooled or heat pipe heat sinks are positioned and windings are wound on it [27].

\section{FUtURE RESEARCH DIRECTION}

Nowadays, the development of high power density and efficient HFT is one of the interesting research areas as the application of HFT has increased significantly in recent years. To achieve a compact and lightweight design, the magnetic core material should have simultaneously higher saturation flux density and lower iron loss. Both properties cannot be obtained simultaneously so far. Therefore, in future the magnetic materials should be developed to achieve both higher saturation flux density and lower specific core loss. Due to non-sinusoidal input voltage, the core loss prediction becomes difficult. Simple and accurate model can be developed in future for easier core loss prediction. Efforts can be also emphasized to easy and accurate modeling for other parameters of transformer, e.g. leakage inductance, resistance, and stray capacitance. In addition, generalized optimization techniques can be developed to acquire more global optimal design. Finally, the low volume, low weight and high efficiency HFT's design is always the attractive research focus for machine designers. Beside the development of HFT, the development of high-frequency common magnetic-link or bus with advanced magnetic materials can be investigated further to integrate multiple power sources magnetically, i.e. magnetic synchronization which is not yet discussed in literature. As the HFT usually excites through a power electronic circuit, the limitations and properties of power switching devices should be well investigated during the characterization of magnetic materials. Recent development of silicon carbide ( $\mathrm{SiC}$ ) switching devices could be a new route of research in designing HFT. HTS technology can be combined with the advanced magnetic material based HFT technology to reduce the size and weight of the transformer further and improve the performance as well.

\section{CONCLUSION}

This paper presents an elaboration of different elements of HFTs which can directly affect the design process to help the machine designers to select the core material from broad range of magnetic materials to meet the requirements. To design an HFT, the prediction of iron loss and parasitic elements is necessary. Therefore, a complete survey on different modeling of core loss, leakage resistance, leakage inductance and stray capacitance can help to predict their values to achieve an optimal design. The present and future research directions are also discussed to achieve compact and lightweight HFTs.

\section{REFERENCES}

[1] J. X. Jin, et al., "Enabling high-temperature superconducting technologies toward practical applications," IEEE Trans. Appl. Supercond., vol. 24, no. 5, Oct. 2014, Art. no. 5400712.

[2] M. R. Islam, Y. G. Guo, J. G. Zhu, H. Y. Lu, and J. X. Jin, "Highfrequency magnetic-link medium-voltage converter for superconducting generator-base high power density wind generation systems," IEEE Trans. Appl. Supercond., vol. 25, no. 5, Oct. 2014, Art. no. 5202605.

[3] J. X. Jin, et al., "HTS power devices and systems: principles, characteristics, performance, and efficiency," IEEE Trans. Appl. Supercond., vol. 26, no. 7, Oct. 2016, Art. no. 3800526.

[4] J. S. Jeong, D. K. An, J. P. Hong, H. J. Kim, and Y. S. Jo, "Design of a 10-MW-class HTS homopolar generator for wind turbines," IEEE Trans. Appl. Supercond., vol. 27, no. 4, Jun. 2017, Art. no. 5202804.

[5] M. Saruwatari, et al., "Design study of 15-MW fully superconducting generators for offshore wind turbine," IEEE Trans. Appl. Supercond., vol. 26, no. 4, Jun. 2016, Art. no. 5206805.

[6] Z. Deng et al., "A high-temperature superconducting maglev ring test line developed in Chengdu, China," IEEE Trans. Appl. Supercond., vol. 26, no. 6, Sept. 2016, Art. no. 3602408

[7] Z. H. Chen, J. X. Jin, L. H. Zheng, and Z. H. Wu, "Advanced HTS dc transmission with self-protection function," IEEE Trans. Appl. Supercond., vol. 26, no. 7, Oct. 2016, Art. no. 5403105.

[8] D. I. Doukas, A. Syrpas, and D. P. Labridis, "Multiterminal dc transmission systems based on superconducting cables feasibility study, modeling, and control," IEEE Trans. Appl. Supercond., vol. 28, no. 4, Jun. 2018, Art. no. 5400506

[9] Z. Deng, J. Li, W. Zhang, Y. Gou, Y. Ren, and J. Zheng, "Hightemperature superconducting magnetic levitation vehicles: dynamic characteristics while running on a ring test line," IEEE Veh. Tech. Magazine, vol. 12, no. 3, pp. 95-102, Sept. 2017.

[10] M. R. Islam, Y. G. Guo, and J. G. Zhu, "A multilevel medium-voltage inverter for step-up-transformer-less grid connection of photovoltaic power plants," IEEE J. Photovoltaics, vol. 4, no. 3, pp. 881-889, May 2014

[11] M. R. Islam, Y. G. Guo, and J. G. Zhu, "A medium-frequency transformer with multiple secondary windings for medium-voltage converter based wind turbine generating systems," J. Appl. Physics, vol. 113, no. 17, May 2013, Art. no. 17A324.

[12] X. She, A. Q. Huang, and R. Burgos, "Review of solid-state transformer technologies and their application in power distribution systems," IEEE J. Emer. Sele. Topics Power Electron., vol. 1, no. 3, pp. 186-198, Sep. 2013

[13] S. Zhao, Q. Li, F. C. Lee, and B. Li, "High-frequency transformer design for modular power conversion from medium-voltage ac to 400 Vdc," IEEE Trans. Power Electron, vol. 33, no. 9, pp. 7545-7557, Sept. 2018

[14] L. Zhang, D. Zhang, H. Shui, Y. Yuan, Q. Pei, and J. Zhu, "Optimisation design of medium frequency transformer for the offshore dc grid based on multi-objective genetic algorithm," IET Power Electronics, vol. 10, no. 15, pp. 2157-2162, Dec. 2017.

[15] M. Jafari, Z. Malekjamshidi, G. Lei, T. Wang, G. Platt, and J. Zhu, "Design and implementation of an amorphous high-frequency transformer coupling multiple converters in a smart microgrid," IEEE Trans. Ind. Electron, vol. 64, no. 2, pp. 1028-1037, Feb. 2017.

[16] A. Krings, A. Boglietti, A. Cavagnino, and S. Sprague, "Soft magnetic material status and trends in electric machines," IEEE Trans. Ind. Electron, vol. 64, no. 3, pp. 2405-2414, Mar. 2017.

[17] M. A. Bahmani, "Design and optimization considerations of mediumfrequency power transformers in high-power dc-dc applications," Thesis Dissertation, Chalmers University of Technology Gothenburg, Sweden, 2016.

[18] C. Xiao, G. Chen, and W. G. H. Odendaal, "Overview of power loss measurement techniques in power electronic systems," IEEE Trans. Ind. Appl, vol. 43, no 3, pp 657-664, May-Jun. 2007.

[19] D. K. Conroy and R. F. Cascio, "Measurement techniques for the design of high-frequency SMPS transformer," in Proc. 3rd Annu. Meeting Appl. Power Electron. Conf. Expo., New Orleans, LA, USA, Feb. 1-5, 1988, pp. 633-640. 
[20] M. Mu, Q. Li, D. J. Gilham, F. C. Lee, and K. D. T. Ngo, "New core loss measurement method for high-frequency magnetic materials," IEEE Trans. Power Electron., vol. 29, no. 8, pp. 4374-4381, Aug. 2014.

[21] I. Villar, U. Viscarret, I. Etxeberria-Otadui, and A. Rufer, "Global loss evaluation methods for nonsinusoidally fed medium-frequency power transformers," IEEE Trans. Ind. Electron, vol. 56, no. 10, pp. 4132-4140, Oct. 2009.

[22] S. Barg, K. Ammous, H. Mejbri, and A. Ammous, "An improved empirical formulation for magnetic core losses estimation under nonsinusoidal induction," IEEE Trans. Power Electron, vol. 32, no. 3, pp. 2146-2154, Mar. 2017.

[23] P. L. Dowell, "Effects of eddy currents in transformer windings, " in Proc. Inst. Elec. Engr., vol. 113, no. 8, pp. 1387-1394, Aug. 1966.

[24] A. Reatti and M. K. Kazimierczuk, "Comparison of various methods for calculating the ac resistance of inductors," IEEE Trans. Magn., vol. 38, no. 3, pp. 1512-1518, May 2002.

[25] Z. Ouyang, J. Zhang, and W. G. Hurley, "Calculation of leakage inductance for high-frequency transformers," IEEE Trans. Power Electron, vol. 30, no. 10, pp. 5769-5775, Oct. 2015.

[26] N. B. Chagas and T. B. Marchesan, "Analytical calculation of static capacitance for high frequency inductors and transformers," IEEE Trans. Power Electron, in press, doi: 10.1109/TPEL.2018.2829716.

[27] G. Ortiz, J. Biela, and J. W. Kolar, "Optimized design of medium frequency transformers with high isolation requirements," in Proc. 36th Annu. Conf. IEEE Ind. Electron. Soc. IECON, Glendale, AZ, Nov. 7-10, 2010, pp. 631-638.

[28] M. Pavlovsky, S. W. H. de Haan, and J. A. Ferreira, "Design for better thermal management in high-power high-frequency transformers," in Proc. 40th IAS Annu. Meeting. Conf. Industry Appl., Kowloon, Hong Kong, Oct. 2-3, 2005, vol. 4, pp. 2615-2621.

[29] O. de la Barrière, C. Ragusa, C. Appino, and F. Fiorillo, "Prediction of energy losses in soft magnetic materials under arbitrary induction waveforms and dc bias," IEEE Trans. Ind. Electron, vol. 64, no. 3, pp. 2522-2529, Mar. 2017.

[30] P. Shuai and J. Biela, "Influence of material properties and geometric shape of magnetic cores on acoustic noise emission of medium-frequency transformers," IEEE Trans. Power Electron, vol. 32, no. 10, pp. 79167931, Oct. 2017.

[31] M. Leibl, G. Ortiz, and J. W. Kolar, "Design and experimental analysis of a medium-frequency transformer for solid-state transformer applications," IEEE J. Emer. Sele. Topics Power Electron., vol. 5, no. 1, pp. 110-123, Mar. 2017.

[32] M. R. Islam, K. M. Muttaqi, D. Sutanto, and J. Zhu, "Design and implementation of amorphous magnetic material common magnetic bus for the replacement of common dc bus," IEEE Trans. Magn., to be published, doi: 10.1109/TMAG.2018.2844334.

[33] Metglas, Magnetic Alloy, Technical Bulletin. Accessed: Jul. 10, 2018. [Online]. Available: https://metglas.com/

[34] K. Inagaki, et al., "Amorphous Transformer Contributing to Global Environmental Protection," Hitachi Review, vol. 60, no 5, 2011.

[35] Vacuumschelze GmbH \& Co. KG. Properties of Amorphous Co-based Alloys with F-characteristics. Accessed: Jul. 10, 2018. [Online]. Available: https://www.vacuumschmelze.com/

[36] M. Ohta and R. Hasegawa, "Soft magnetic properties of magnetic cores assembled with a high $\mathrm{B}_{\mathrm{s}}$ Fe-based nanocrystalline alloy," IEEE Trans. Magn., vol. 53, no. 2, Feb. 2017, Art no. 2000205.

[37] M. Ohta, R. Hasegawa, and H. Itabashi, "Development of block cores comprising high- $B_{s}$ nanocrystalline alloy ribbon," IEEE Trans. Magn, vol. 54, no. 5, May 2018, Art no. 2000504

[38] E. Agheb and H. K. Hoidalen, "Modification of empirical core loss calculation methods including flux distribution," IET Electric Power Appl., vol. 7, no. 5, pp. 381-390, May 2013.

[39] S. A. Mousavi, G. Engdahl, and E Agheb, "Investigation of GIC effects on core losses in single phase power transformers," Arch. Elect. Engg., vol. 60 , no.1, pp. 35-47, 2011.

[40] C. P. Steinmetz, "On the law of hysteresis," Trans. American Ins. Elect. Engr., no. 1, pp. 1-64, Jan. 1892.

[41] J. Reinert, A. Brockmeyer, and R. W. A. A. De Doncker, "Calculation of losses in ferro- and ferrimagnetic materials based on the modified Steinmetz equation," IEEE Trans. Ind. Appl, vol. 37, no. 4, pp. 10551061, Jul.-Aug. 2001.

[42] J. Li, T. Abdallah, and C. R. Sullivan, "Improved calculation of core loss with nonsinusoidal waveforms," in Proc. IEEE Ind. Appl Conf. 36th IAS Annu Meeting, Chicago, IL, USA, Sep. 30-04 Aug. 2001, vol. 4, pp. 2203-2210.
[43] K. Venkatachalam, C. R. Sullivan, T. Abdallah, and H. Tacca, "Accurate prediction of ferrite core loss with nonsinusoidal waveforms using only Steinmetz parameters," in Proc. IEEE Workshop Comp. Power Electron., Mayaguez, Puerto Rico, USA, Jun. 3-4, 2002, pp. 36-41.

[44] A. Van den Bossche, V. C. Valchev, and G. B. Georgiev, "Measurement and loss model of ferrites with non-sinusoidal waveforms, " in Proc. IEEE 35th Annu. Power Electron. Spec. Conf., Aachen, Germany, Jun. 20-25, 2004, vol. 6, pp. 4814-4818.

[45] W. Shen, F. Wang, D. Boroyevich, and C. W. Tipton, "Loss characterization and calculation of nanocrystalline cores for highfrequency magnetics applications," IEEE Trans. Power Electron, vol. 23, no. 1, pp. 475-484, Jan. 2008.

[46] J. Muhlethaler, J. Biela, J. W. Kolar, and A. Ecklebe, "Improved core-loss calculation for magnetic components employed in power electronic systems," IEEE Trans. Power Electron, vol. 27, no. 2, pp. 964-973, Feb. 2012.

[47] M. R. Islam, Y. G. Guo, and J. G. Zhu, "An amorphous alloy core medium frequency magnetic-link for medium voltage photovoltaic inverters," $J$. Appl. Physics, vol. 115, no. 17, May 2014, Art. no. 17E710.

[48] M. R. Islam, Y. G. Guo, and J. G. Zhu, "Power converters for medium voltage networks," Green Energy and Technology Series, SpringerVerlag GmbH, Heidelberg, Germany, Sept. 2014.

[49] M. Mu, "High frequency magnetic core loss study," PhD Thesis Dissertation, Virginia Polytechnic Institute and State University, USA, 2013.

[50] R. Linkous, A. W. Kelley, and K. C. Armstrong, "An improved calorimeter for measuring the core loss of magnetic materials," in Proc. 15th Annu. IEEE Applied Power Electron. Conf. Exp., New Orleans, LA, Feb. 6-10, 2000, vol. 2, pp. 633-639.

[51] M. R. Islam, G. Lei, Y. Guo, and J. Zhu, "Optimal design of highfrequency magnetic links for power converters used in grid-connected renewable energy systems," IEEE Trans. Magn, vol. 50, no. 11, Nov. 2014, Art no. 2006204.

[52] Y. Han and Y. F. Liu, "A practical transformer core loss measurement scheme for high-frequency power converter," IEEE Trans. Ind. Electron., vol. 55, no. 2, pp. 941-948, Feb. 2008.

[53] Y. Han, W. Eberle, and Y. F. Liu, "New measurement methods to characterize transformer core loss and copper loss in high frequency switching mode power supplies," in Proc. IEEE 35th Annu. Power Electron. Spec. Conf., Aachen, Germany, Jun. 20-25, 2004, vol.2, pp. 1695-1701.

[54] M. Mu, Q. Li, D. Gilham, F. C. Lee, and K. D. T. Ngo, "New core loss measurement method for high frequency magnetic materials," in Proc. IEEE Energy Conv. Cong. Expo., Atlanta, GA, Sept. 12-16, 2010, pp. 4384-4389.

[55] M. Mu, F. C. Lee, Q. Li, D. Gilham, and K. D. T. Ngo, "A high frequency core loss measurement method for arbitrary excitations," in Proc. IEEE 26 Annu. Appl. Power Electron. Conf. Expo., Fort Worth, TX, Mar. 6-11, 2011, pp. 157-162.

[56] D. Hou, M. Mu, F. C. Lee, and Q. Li, "New high-frequency core loss measurement method with partial cancellation concept," IEEE Trans. Power Electron, vol. 32, no. 4, pp. 2987-2994, Apr. 2017.

[57] W. G. Hurley, E. Gath, and J. G. Breslin, "Optimizing the ac resistance of multilayer transformer windings with arbitrary current waveforms," IEEE Trans. Power Electron, vol. 15, no. 2, pp. 369-376, Mar 2000.

[58] M. P. Perry, "Multiple layer series connected winding design for minimum losses," IEEE Trans. Power App. Systems, vol. PAS-98, no. 1, pp. 116-123, Jan. 1979.

[59] J. A. Ferreira, "Appropriate modelling of conductive losses in the design of magnetic components," in Proc. 21st Annual IEEE Conf. Power Electron. Spec., San Antonio, TX, USA, 1990, pp. 780-785.

[60] J. A. Ferreira, "Analytical computation of AC resistance of round and rectangular Litz wire windings," in Proc.-B IEE Electric Power Appl., Jan. 1992. vol. 139, no. 1, pp. 21-25.

[61] M. Bartoli, N. Noferi, A. Reatti, and M. K. Kazimierczuk, "Modeling winding losses in high frequency power inductors," World Sc. J. Circ., Syst. Comp., Special Issue Power Electronics, part II, vol. 5, pp. 607-626, Dec. 1996.

[62] M. A. Bahmani and T. Thiringer, "Accurate evaluation of leakage inductance in high-frequency transformers using an improved frequencydependent expression," IEEE Trans. Power Electron, vol. 30, no. 10, pp. 5738-5745, Oct. 2015.

[63] A. Stadler and M. Albach, "The influence of the winding layout on the core losses and the leakage inductance in high frequency transformers," IEEE Trans. Magn, vol. 42, no. 4, pp. 735-738, Apr. 2006. 
[64] A. Massarini and M. K. Kazimierczzuk, "Self-capacitance of inductor," IEEE Trans. Power Electron, vol. 12, no. 4, pp. 671-676, Jul. 1997.

[65] L. d'Alessandro, F. da Silveira Cavalcante, and J. W. Kola, "Self capacitance of high voltage transformer," IEEE Trans. Power Electron, vol. 22, no. 5, pp. 2081-2092, Sept. 2007.

[66] X. Liu, Y. Wang, J. Zhu, Y. Guo, G. Lei, and C. Liu, "Calculation of capacitance in high-frequency transformer windings," IEEE Trans. Magn, vol. 52, no. 7, Jul. 2016, Art. no. 2003204.

[67] M. A. Bahmani, T. Thiringer, and M. Kharezy, "Design methodology and optimization of a medium-frequency transformer for high-power dc-dc applications," IEEE Trans. Ind. Appl, vol. 52, no. 5, pp. 4225-4233, Sept.Oct. 2016.

[68] J. Li, W. Water, B. Zhu, and J. Lu, "Integrated high-frequency coaxial transformer design platform using artificial neural network optimization and FEM simulation," IEEE Trans. Magn, vol. 51, no. 3, March 2015, Art. no. 8500204.

[69] L. Zhang, D. Zhang, H. Shui, Y. Yuan, Q. Pei, and J. Zhu, "Optimisation design of medium frequency transformer for the offshore dc grid based on multi-objective genetic algorithm," IET Power Electron., vol. 10, no. 15, pp. 2157-2162, 2017.

[70] M. Leibl, G. Ortiz, and J. W. Kolar, "Design and experimental analysis of a medium-frequency transformer for solid-state transformer applications," IEEE J. Emer. Sele. Topics Power Electron., vol. 5, no. 1, pp. 110-123, March 2017.

[71] M. R. Islam, A. M. M. Rahman, M. M. Islam, Y. G. Guo, and J. G. Zhu, "A modular medium voltage grid connected converter with improved switching techniques for solar photovoltaic systems," IEEE Trans. Ind. Electron., vol. 64, no. 11, pp. 8887-8896, Nov. 2017.

[72] M. R. Islam, Y. G. Guo, and J. G. Zhu, "A high-frequency link multilevel cascaded medium-voltage converter for direct grid integration of renewable energy systems," IEEE Trans. Power Electron., vol. 29, no. 8, pp. 4167-4182, Aug. 2014.

[73] G. B. Kumbhar and S. V. Kulkarni, "Analysis of short-circuit performance of split-winding transformer using coupled field-circuit approach," IEEE Trans. Power Deli., vol. 22, no. 2, pp. 936-943, Apr. 2007.

[74] P. Sun and J. Huang, "The finite element analysis of the leakage inductance for high frequency switching mode power supply transformer," Adv. Mate. Res., vol. 614-615, pp. 1122-1125, 2013.

[75] E. L. Barrios, A. Ursúa, L. Marroyo, and P. Sanchis, "Analytical design methodology for Litz-wired high-frequency power transformers," IEEE Trans. Ind. Electron., vol. 62, no. 4, pp. 2103-2113, Apr. 2015. 